\title{
Lysophosphatidic acid impairs glucose homeostasis and inhibits insulin secretion in high-fat diet obese mice
}

\author{
C. Rancoule • C. Attané • S. Grès • A. Fournel • \\ R. Dusaulcy $\cdot$ C. Bertrand $\cdot$ C. Vinel $\cdot$ K. Tréguer . \\ M. Prentki • P. Valet • J. S. Saulnier-Blache
}

Received: 3 October 2012 / Accepted: 4 March 2013 /Published online: 19 March 2013

(C) Springer-Verlag Berlin Heidelberg 2013

\begin{abstract}
Aims/hypothesis Lysophosphatidic acid (LPA) is a lipid mediator produced by adipocytes that acts via specific Gprotein-coupled receptors; its synthesis is modulated in obesity. We previously reported that reducing adipocyte LPA production in high-fat diet (HFD)-fed obese mice is associated with improved glucose tolerance, suggesting a negative impact of LPA on glucose homeostasis. Here, our aim was to test this hypothesis.

Methods First, glucose tolerance and plasma insulin were assessed after acute (30 min) injection of LPA $(50 \mathrm{mg} / \mathrm{kg})$ or of the LPA1/LPA3 receptor antagonist Ki16425 (5 $\mathrm{mg} \mathrm{kg}^{-1}$ day $^{-1}$, i.p.) in non-obese mice fed a normal diet (ND) and in obese/prediabetic (defined as glucose-intolerant) HFD mice. Glucose and insulin tolerance, pancreas morphology,
\end{abstract}

Electronic supplementary material The online version of this article (doi:10.1007/s00125-013-2891-3) contains peer-reviewed but unedited supplementary material, which is available to authorised users.

C. Rancoule $\cdot \mathrm{S}$. Grès $\cdot$ A. Fournel $\cdot$ R. Dusaulcy $\cdot$ C. Bertrand $\cdot$

C. Vinel $\cdot$ K. Tréguer $\cdot$ P. Valet $\cdot$ J. S. Saulnier-Blache $(\triangle)$

Institut des Maladies Métaboliques et Cardiovasculaires (I2MC),

Université Paul Sabaties, Inserm U1048, 1 avenue Jean Poulhès,

BP 84225, 31432 Toulouse Cedex 4, France

e-mail: Jean-Sebastien.Saulnier-Blache@inserm.fr

C. Attané $\cdot$ M. Prentki

Molecular Nutrition Unit and Montreal Diabetes Research Center at the Centre de Recherche du Centre Hospitalier de l'Université de Montréal (CHUM), Montréal, QC, Canada

M. Prentki

Department of Nutrition, Université de Montréal,

Montréal, QC, Canada

M. Prentki

Department of Biochemistry, Université de Montréal,

Montréal, QC, Canada glycogen storage, glucose oxidation and glucose transport were then studied after chronic treatment ( 3 weeks) of HFD mice with Ki16425.

Results In ND and HFD mice, LPA acutely impaired glucose tolerance by inhibiting glucose-induced insulin secretion. These effects were blocked by pre-injection of $\mathrm{Ki16425}$ (5 mg/kg, i.p.). Inhibition of glucoseinduced insulin secretion by LPA also occurred in isolated mouse islets. Plasma LPA was higher in HFD mice than in ND mice and Ki16425 transiently improved glucose tolerance. The beneficial effect of Ki16425 became permanent after chronic treatment and was associated with increased pancreatic islet mass and higher fasting insulinaemia. Chronic treatment with Ki16425 also improved insulin tolerance and increased liver glycogen storage and basal glucose use in skeletal muscle.

Conclusions/interpretation Exogenous and endogenous LPA exerts a deleterious effect on glucose disposal through a reduction of plasma insulin; pharmacological blockade of LPA receptors improves glucose homeostasis in obese/prediabetic mice.

Keywords Antagonist · Glucose homeostasis · Insulin · Lysophosphatidic acid · Obesity $\cdot$ Receptors

\begin{tabular}{ll}
\multicolumn{2}{l}{ Abbreviations } \\
ATX & Autotaxin \\
FATX-KO & Adipocyte-specific ATX knockout \\
HFD & High-fat diet \\
KH & Krebs-Henseleit \\
KRBH & Krebs Ringer Bicarbonate HEPES buffer \\
LPA & Lysophosphatidic acid \\
MCP & Monocyte chemotactic protein \\
ND & Normal diet
\end{tabular}




\section{Introduction}

Excessive accumulation of adipose tissue in obesity is frequently associated with metabolic defects (postprandial hyperglycaemia, dyslipidaemia, insulin resistance) that may lead to type 2 diabetes and cardiovascular diseases (hypertension, atherosclerosis, nephropathies) $[1,2]$. In parallel with its lipid storage capacity, the adipose tissue produces various bioactive molecules (adipokines, reactive phospholipids, glycerol, NEFA). The export of these molecules from adipose tissue is often altered in obese individuals and some of them (TNF- $\alpha$, resistin, monocyte chemotactic protein-1 [MCP-1]) exert deleterious effects on glucose metabolism and insulin sensitivity [3].

Lysophosphatidic acid (LPA) is a phospholipid mediator that regulates several cellular responses (proliferation, migration, differentiation) via specific G-protein-coupled receptors (LPA1R to LPA6R) [4]. LPA is synthesised via the hydrolysis of lysophosphatidylcholine by a secreted lysophospholipase D (autotaxin [ATX]) [5, 6]. The available pharmacological tools targeting ATX/LPA signalling are still scarce [7-9]. Ki16425 [10] is a mixed LPA receptor antagonist that exhibits close and preferential affinity for the LPA1R and LPA3R subtypes (250 and $360 \mathrm{nmol} / \mathrm{l}$, respectively) $[8,10]$ and its in vivo antagonist efficiency has been documented [11-13].

White adipose tissue secretes ATX and LPA in the extracellular milieu $[12,14-16]$. The expression of ATX is increased in the adipose tissue of obese insulin-resistant individuals and mice [16-18]. Plasma levels of LPA are increased in high-fat diet (HFD) obese mice as the result of an increased expression of ATX in adipocytes [19]. Invalidation of ATX in adipocytes (FATX-KO mice) reduces plasma LPA and enhances fat mass in HFD-fed mice [19], in agreement with the anti-adipogenic effect of LPA [20].

Whereas the growth factor-like properties of LPA have been studied extensively, a few and primarily in vitro studies have reported that LPA also regulates glucose metabolism [21-23]. Interestingly, in vivo deletion of ATX in adipocytes (FATX-KO mice) is associated with a better glucose tolerance [19], suggesting a negative effect of LPA on glucose homeostasis. If this hypothesis is correct, LPA receptors could represent valuable pharmacological targets for the treatment of impaired glucose homeostasis associated with obesity.

Our objective was to study the influence of LPA on glucose and insulin homeostasis. We found that administration of LPA to mice impairs glucose disposal via a reduction of plasma insulin levels, and that chronic pharmacological blockade of the LPA receptors restores normal glucose homeostasis in HFD obese/prediabetic (defined as glucose intolerant) mice.

\section{Methods}

Mice C57B16/J male mice were housed conventionally under a constant temperature $\left(20-22^{\circ} \mathrm{C}\right)$ and humidity $(50-60 \%)$ with a $12 / 12 \mathrm{~h}$ light/dark cycle (lights on at 07:00 hours) and free access to food and water. Mice were handled in accordance with the principles and guidelines established by the National Institute of Medical Research (Inserm) and in conformity with the 'Principles of laboratory animal care' (NIH publication no. 85-23, revised 1985 http://grants1.nih.gov/grants/olaw/references/phspol.htm). The local animal facility committee at Inserm approved our protocols.

Diet The mice were first fed a normal diet (ND; energy content $16 \%$ protein, $81 \%$ carbohydrate and $3 \%$ fat [SAFE, Augy, France]) until the age of 10 weeks. Mice were then fed either an ND or an HFD (energy content $20 \%$ protein, $35 \%$ carbohydrate and $45 \%$ fat [Research Diets-Brogaarden (Lynge, Denmark)]) for 9 weeks. At that step, HFD mice were considered obese and prediabetic. They had a higher body weight (35.6 \pm 1.0 vs $29.9 \pm 0.6 \mathrm{~g} ; p<0.01)$ and a higher body-fat mass $(24.6 \pm$ 2.0 vs $5.6 \pm 1.1 \%$ of total body weight; $p<0.001$ ) when compared with ND mice. Moreover, HFD mice displayed a deterioration in glucose tolerance (35\% increase in AUC when compared with ND mice) (Control, Fig. 1d, e vs i, j) associated with a slight increase in fasting glycaemia (Control, Fig. $1 \mathrm{~b}$ vs g) but no change in fasting insulinaemia (Control, Fig. 1c vs h).

Treatment Ki16425 Cayman Chemicals-Interchim (Montluçon, France) was diluted at a concentration of $1 \mathrm{mg} / \mathrm{ml}$ in PBS supplemented with $10 \%$ DMSO. LPA (oleoyl lysophosphatidic acid; Avanti Polar, Alabaster, AL, USA) was diluted at $15 \mathrm{mg} / \mathrm{ml}$ in PBS supplemented with $1 \%$ fatty-acid-free BSA. Ki16425 (5 mg/kg), LPA $(50 \mathrm{mg} / \mathrm{kg}$ ) and vehicle (control) were administered by i.p. injection. Treatments with Ki16425 and LPA started 9 weeks after beginning the feeding protocol. The dose of LPA injected was adapted from previous reports $[24,25]$. The concentration of Ki16425 used had previously been demonstrated to block LPA receptors in vivo [26]. Acute treatments consisted of injecting a single dose of LPA or Ki16425 30 min before conducting glucose or insulin tolerance tests. Acute treatments were performed on ND and HFD mice after 9 weeks of diet. Chronic treatment consisted of a daily injection of Kil6425 for 3 weeks. HFD was maintained during chronic treatment. Chronic treatment was followed by glucose or insulin tolerance tests, which were conducted $24 \mathrm{~h}$ after the last injection of Ki16425. After glucose or insulin tolerance tests, treatment with Ki16425 was continued for few days before the mice were killed after an overnight fasting. Plasma and organs were collected for further analysis. 


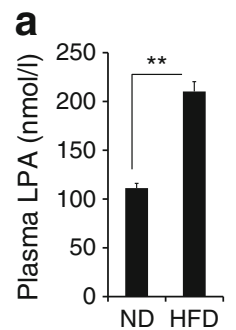

b

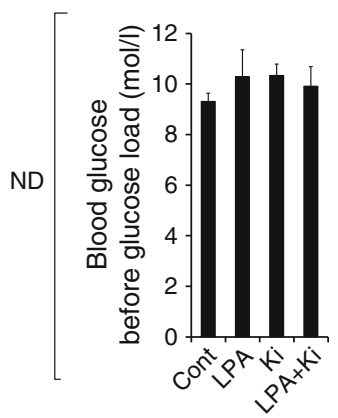

g

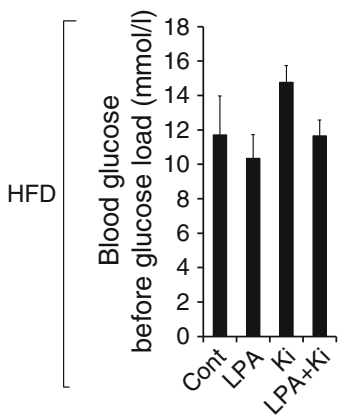

C

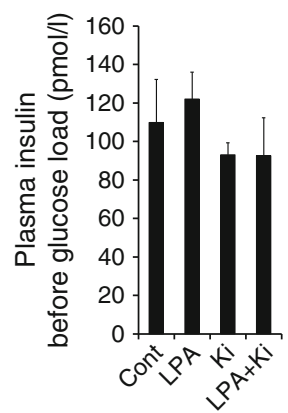

h

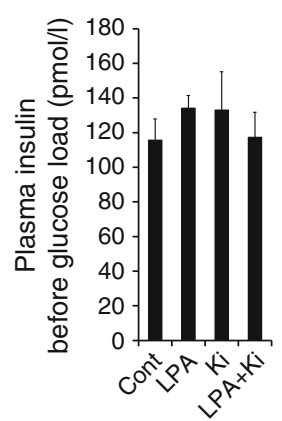

d

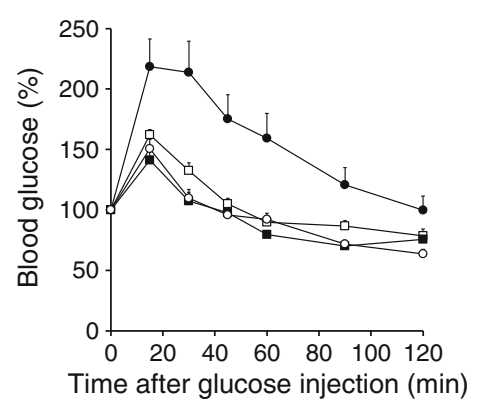

i

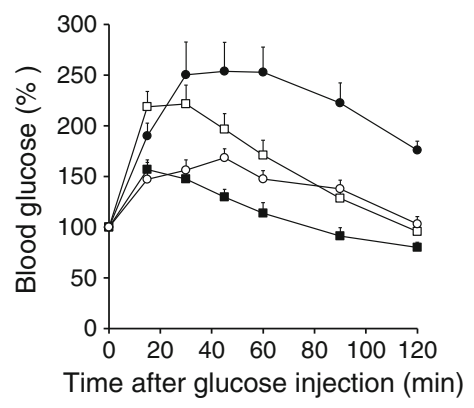

e

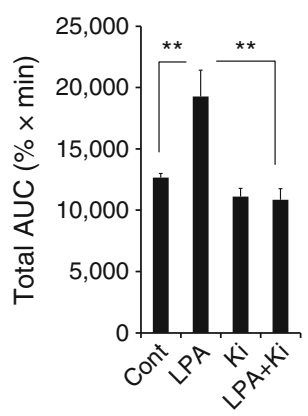

j

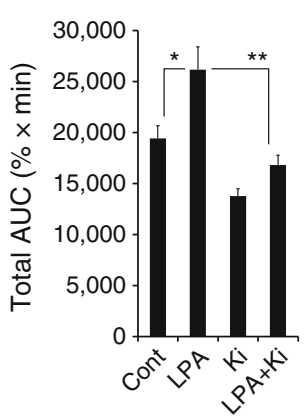

f

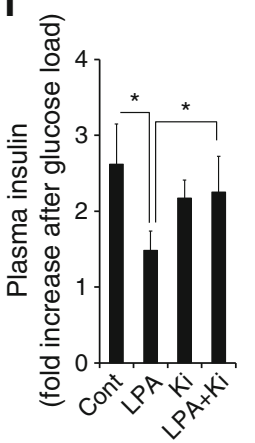

k

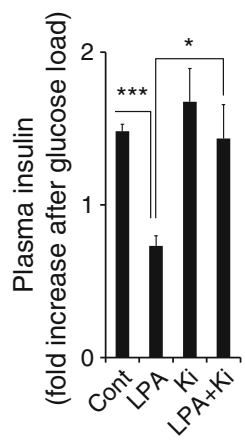

Fig. 1 Acute regulation of glucose tolerance by LPA and Ki16425 in ND and HFD mice. (a) Plasma LPA concentration in ND and HFD mice after 9 weeks of diet. (b-k) $7 \mathrm{~h}$ fasted ND (b-f) and HFD (g-k) mice received a single injection of LPA or vehicle (Cont) with or without a pre-injection of Ki16425 (Ki) administered 10 min before the LPA or vehicle. White squares, vehicle; black circles, LPA; black squares, Ki16425; white circles, LPA + Ki16425. After $30 \mathrm{~min}$ a glucose tolerance test was conducted. Blood glucose (b, g) and plasma insulin $(\mathbf{c}, \mathbf{h})$ concentrations measured before glucose load. (d, i) Blood glucose concentration during the glucose tolerance test, expressed as per cent of concentration at time 0 , and corresponding $\operatorname{AUC}(\mathbf{e}, \mathbf{j})$. (f, $\mathbf{k})$ Fold change in plasma insulin from $15 \mathrm{~min}$ before to $15 \mathrm{~min}$ after glucose load. Values are means \pm SEM of four to six mice per group. ${ }^{*} p<0.05,{ }^{* *} p<0.01$ and ${ }^{* * *} p<0.001$, comparing indicated values
Glucose and insulin tolerance tests Mice were fasted for $7 \mathrm{~h}$ before i.p. injection of glucose $(1 \mathrm{~g} / \mathrm{kg})$ or insulin $(0.75 \mathrm{U} / \mathrm{kg})$. Blood from the tail vein was sampled before glucose load and every $15 \mathrm{~min}$ over $120 \mathrm{~min}$ to monitor glucose concentration. Plasma was collected 15 min before and after glucose injection to quantify insulin concentration. Glucose and insulin tolerance tests were conducted on separate groups of mice to limit the stress on the mice.

Body-fat-mass composition Fat and lean masses were determined by placing mice in a clear plastic holder, without anaesthesia or sedation, and inserting into the EchoMRI-3in-1 system (Echo Medical Systems, Houston, TX, USA). The measure was performed 1 day before acute treatments with LPA and Ki16425, and after chronic treatment with Ki16425.

Blood analysis Blood glucose was quantified using a glucose meter (Accu-Chek; Roche Diagnostic, Grenoble, France). Plasma insulin was quantified using a mouse insulin ELISA kit (Mercodia, Uppsala, Sweden). Plasma NEFA were measured using the enzymatic colorimetric Wako NEFA kit (Wako Chemicals, Neuss, Germany). Plasma triacylglycerols were measured using the enzymatic colorimetric PAP $150 \mathrm{Kit}$ (BioMerieux, Marcy l'Etoile, France). Plasma leptin and adiponectin were determined using Quantkine Immunoassays (R\&D Systems, Minneapolis, MN, USA). Plasma LPA was measured using a radioenzymatic assay as previously 
described [19, 27]. Briefly, lipids were extracted from conditioned media or plasma with an equal volume of 1-butanol and evaporated. Extracted lipids were converted into $\left[{ }^{14} \mathrm{C}\right]$ phosphatidic acid with recombinant LPA acyl-transferase in the presence of $\left[{ }^{14} \mathrm{C}\right]$ oleoyl-CoA. The products of the reaction were extracted, separated by one-dimensional thin-layer chromatography and autoradiographed.

Organ triacylglycerol content Skeletal muscle and liver were homogenised and triacylglycerols were extracted from $500 \mu \mathrm{l}$ homogenate using $2.5 \mathrm{ml}$ chloroform/methanol (2:1 vol./vol.) and $1.2 \mathrm{ml} \mathrm{KCl} / \mathrm{HCl} 2 \mathrm{~mol} / \mathrm{l}$ was added. The organic phase was evaporated and solubilised in isopropyl alcohol, and triacylglycerols were quantified using an enzymatic colorimetric PAP 150 Kit (BioMerieux).

Organ glycogen content Skeletal muscle or liver was digested in $\mathrm{NaOH}(1 \mathrm{~mol} / \mathrm{l})$ and digestion was stopped with $\mathrm{HCl}(1 \mathrm{~mol} / \mathrm{l})$. Digestate was diluted in sodium acetate (1:4 vol./vol.) unsupplemented or supplemented with amyloglucosidase $(500 \mathrm{U} / \mathrm{ml})$ (Sigma-Aldrich, St Quentin Fallavier, France) and incubated at $55^{\circ} \mathrm{C}$ for $1 \mathrm{~h}$ to transform glycogen into glucose. Released glucose was quantified using an RTU kit (BioMerieux).

Glucose oxidation in ex vivo muscle Glucose oxidation was measured as previously described [28] with minor modifications. Red bundles from gastrocnemus muscle were incubated in modified Krebs-Henseleit (KH) buffer containing 0.2\% fatty-acid-free BSA, $5 \mathrm{mmol} / \mathrm{l}$ glucose and $5,550 \mathrm{~Bq} / \mathrm{ml}\left[{ }^{14} \mathrm{C}\right]$ glucose (PerkinElmer, Woodbridge, ON, Canada) for $60 \mathrm{~min}$ at $37^{\circ} \mathrm{C}$ in a sealed glass vial. At the end of the incubation, muscles were removed and homogenised in $800 \mu$ lysis buffer to determine protein quantity. Oxidation was determined by acidifying the incubation buffer with $1 \mathrm{ml}$ of $\mathrm{H}_{2} \mathrm{SO}_{4} 1 \mathrm{~mol} / \mathrm{l}$, and the ${ }^{14} \mathrm{CO}_{2}$ was trapped by benzethonium hydroxide (Sigma-Aldrich) placed in a $0.5 \mathrm{ml}$ microtube in the sealed glass vial. After $180 \mathrm{~min}$, the microtube was removed and placed in a scintillation vial and radioactivity was counted (Cytoscint; MP Biomedicals, Illkirch, France).

Glucose transport in ex vivo muscle Glucose transport was measured as previously described [29]. Soleus muscles were isolated and preincubated for $10 \mathrm{~min}$ in $\mathrm{KH}$ buffer, $\mathrm{pH}$ 7.4, containing BSA $(2 \mathrm{mg} / \mathrm{ml}), 2 \mathrm{mmol} / \mathrm{l}$ sodium pyruvate and $20 \mathrm{mmol} / 1$ HEPES. Muscles were then incubated for $45 \mathrm{~min}$ in the absence or the presence of insulin $(100 \mathrm{nmol} / \mathrm{l})$. For glucose transport, muscles were transferred into another vial containing KH medium supplemented with insulin, D-2deoxyglucose $(0.1 \mathrm{mmol} / \mathrm{l})$ and 2-deoxy-D- $\left[{ }^{3} \mathrm{H}\right] \mathrm{glucose}$ $(14,800 \mathrm{~Bq} / \mathrm{ml})$ for $10 \mathrm{~min}$. Muscles were then washed for $1 \mathrm{~h}$ in ice-cold PBS and dissolved in $1 \mathrm{~mol} / \mathrm{l} \mathrm{NaOH}$ for a period of 1 h. 2-Deoxy-D- $\left[{ }^{3} \mathrm{H}\right]$ glucose 6-phosphate and
2-deoxy-D- $\left[{ }^{3} \mathrm{H}\right]$ glucose were differentially precipitated by the use of zinc sulfate $(0.3 \mathrm{~mol} / \mathrm{l})$, barium hydroxide $(0.3 \mathrm{~mol} / \mathrm{l})$ and perchloric acid solutions $(6 \%)$. Radioactivity of each precipitate was quantified in a liquid scintillation fluid.

Insulin secretion from isolated islets Insulin secretion from isolated islets was analysed as previously described [30]. Briefly, islets were isolated from mouse pancreas by collagenase digestion and separated from exocrine tissue by Histopaque gradient. Handpicked islets were placed at $37^{\circ} \mathrm{C}$ in RPMI medium containing $11 \mathrm{mmol} / \mathrm{l}$ glucose for overnight recovery and then distributed in 24-well plates (ten islets per well) for a starvation period of $2 \mathrm{~h}$ in RPMI containing $3 \mathrm{mmol} / \mathrm{l}$ glucose. Islets were then transferred into Krebs Ringer Bicarbonate HEPES buffer (KRBH) containing $3 \mathrm{mmol} / \mathrm{l}$ glucose and $0.07 \%$ BSA for a preincubation period of $45 \mathrm{~min}$ and then incubated for $1 \mathrm{~h}$ in KRBH medium supplemented with $0.5 \%$ BSA containing 3,8 or $16 \mathrm{mmol} / \mathrm{l}$ glucose or $35 \mathrm{mmol} / \mathrm{K} \mathrm{KCl}$ in the absence or presence of LPA $(0.1,1$ and $5 \mu \mathrm{mol} / \mathrm{l})$. Notice that incubation with $\mathrm{KCl}$ allows the measurement of insulin secretion due to complete depolarisation of the islets. The insulin released in incubation buffers was quantified using an AlphaLISA Human Insulin Research Immunoassay Kit (PerkinElmer) and human standards at the Imaging and Cell Biology Core Facility of the Centre de Recherche du CHUM. Total islet insulin content was measured after acid-ethanol $(1.5 \% \mathrm{HCl}, 75 \%$ ethanol $)$ extraction.

Histological analysis of pancreas Immediately after dissection, a piece of pancreas was fixed for $24 \mathrm{~h}$ in formalin $4 \%$, then maintained at $4^{\circ} \mathrm{C}$ in $70 \%$ ethanol until paraffin embedding and sectioned into sections, $5 \mu \mathrm{m}$ thick. Sections were stained with haematoxylin and eosin. An image of the entire slice was performed with NanoZoomer Digital Pathology Image (Hamamatsu [v1.2], Hamamatsu City, Japan) and then analysed with NDP scan 2.5.80 from Hamamatsu to count islet number and the number of cells per islet, and to measure islet area and the total analysed sample area.

$m R N A$ quantification Total RNAs were extracted from tissues and cells using the RNeasy mini kit (Qiagen, Hilden, Germany). Total RNA (500 ng) was reverse transcribed for $60 \mathrm{~min}$ at $37^{\circ} \mathrm{C}$ using Superscript II reverse transcriptase (Invitrogen, St Aubin, France) in the presence of random hexamers. A minus reverse transcriptase (RT) reaction was performed in parallel to ensure the absence of genomic DNA contamination. Real-time PCR was performed on $12.5 \mathrm{ng}$ cDNA and 100-900 nmol/1 specific oligonucleotide primers in a final volume of $20 \mu \mathrm{l}$ using the Mesa blue QPCR Master Mix for Sybr (Eurogentec, Angers, France). Fluorescence was monitored and analysed in a StepOnePlus Real-Time PCR system instrument (Applied Biosystems, St Aubin, France). 
18S RNA was used to normalise gene expression according to the following formula $2^{(\mathrm{Ct} 18 \mathrm{~S}-\mathrm{Ctgene})}$ where $\mathrm{Ct}$ corresponds to the number of cycles needed to generate a fluorescent signal above a predefined threshold. Oligonucleotide primers were designed using the Primer Express software (Applied Biosystems). The sequence of the oligonucleotide primers is listed in electronic supplementary material [ESM] Table 1.

Statistics Data are presented as means \pm SEM. Bilateral Student's $t$ tests were used to compare two groups of data. A two-way ANOVA was applied to detect interaction between treatment and time. Differences were considered significant at $p<0.05$.

\section{Results}

LPA acutely impairs glucose disposal through inhibition of glucose-induced insulin secretion After 9 weeks of diet, HFD mice were obese and prediabetic (see Methods). HFD mice also showed a significantly higher (1.9-fold) plasma LPA concentration than ND fed mice (Fig. 1a). This was in agreement with our previous report [19]. To investigate the possible involvement of LPA in the glucose intolerance of HFD mice, glucose tolerance tests were performed 30 min after a single injection of LPA. In both ND and HFD mice, LPA did not modify basal glycaemia (Cont vs LPA, Fig. 1b vs g) and insulinaemia (Cont vs LPA Fig. 1c vs h) measured before glucose load. This indicated that LPA had no direct impact on these variables. Nevertheless, LPA potently impaired glucose tolerance when compared with control (Cont vs LPA, Fig. 1d, e and i, j). Pre-injection of Ki16425, 10 min before LPA, completely blocked the deleterious effect of LPA on glucose tolerance (LPA vs LPA + Ki, Fig. 1d, e and i, j). These data revealed that exogenous LPA exerts a deleterious effect on glucose tolerance through an LPA-receptor-dependent mechanism.

Interestingly, whereas injection of Ki16425 alone did not significantly influence glucose tolerance in ND mice (Cont vs Ki, Fig. 1d, e), it significantly improved glucose tolerance in HFD mice (Cont vs Ki, Fig. 1i, j). This specific beneficial effect of Ki16425 was compared with the higher concentration of plasma LPA in HFD mice than in ND mice (Fig. 1a). These data strongly suggested that enhanced endogenous production of LPA in HFD mice participates in their impaired glucose tolerance.

In an attempt to understand the mechanisms responsible for LPA-mediated impairment of glucose tolerance, plasma insulin was measured during the glucose tolerance test. In both ND and HFD mice, LPA significantly attenuated the induction of insulinaemia generated by the glucose bolus (Cont vs LPA Fig. 1f, k). LPA-mediated inhibition of plasma insulin was completely blocked by pre-injection of Ki16425
(LPA vs LPA + Ki, Fig. 1f, k). In parallel, insulin tolerance was not significantly altered after injection of LPA or Ki16425 (ESM Fig. 1), suggesting no impact on insulin sensitivity. These data demonstrated that LPA-induced impairment of glucose tolerance resulted from inhibition of glucose-induced insulin secretion rather than alteration of insulin sensitivity. In vitro, LPA dose-dependently inhibited glucose-induced insulin secretion by isolated mouse islets (ESM Fig. 2). In contrast, LPA had no influence on basal insulin secretion or on depolarisation-induced insulin secretion by $\mathrm{KCl}$ (ESM Fig. 2). A direct impact of LPA on the islets could thus be responsible for its inhibitory influence on glucose-induced insulinaemia.

Chronic treatment with Kil6425 improves glucose tolerance in HFD mice We then investigated whether the beneficial effect of Ki16425 on glucose homeostasis in HFD mice could persist over time. In contrast to the observations made after $30 \mathrm{~min}$, at $24 \mathrm{~h}$ after a single injection of Ki16425 glucose tolerance was no more improved when compared with control (ESM Fig. 3). This showed that the beneficial effect of a single injection of Ki16425 on glucose tolerance was transient.

We then examined the effect of chronic treatment with Ki16425 (3 weeks of daily injections). Such treatment had no significant influence on body weight, fat mass or adipose tissue weight (Table 1). Ki16425 treatment had no influence on basal glycaemia preceding the glucose tolerance test (Fig. 2a) but a slight increase in basal insulinaemia was noticed (although the difference did not reach significance) (Fig. 2b). Interestingly, glucose tolerance tests revealed that Ki16425-treatment significantly improved glucose tolerance when compared with control (Fig. 2c, d). This was associated with no significant change in glucose-induced increase in plasma insulin (Fig. 2e). After overnight fasting, Ki16425treated mice showed no significant change in glycaemia (Fig. 2f) but insulinaemia was significantly higher than in control mice (Fig. 2g). These results showed that chronic treatment with Ki16425 persistently improved the glucose tolerance of HFD mice. This beneficial effect of Ki16425 had no consequence on fasting glycaemia but was surprisingly associated with an increase in fasting insulinaemia, suggesting a possible impact on pancreas function.

To test this hypothesis, the pancreas was examined. Histological analysis of the pancreas from Ki16425-treated mice revealed a significantly higher number of islets when compared with control mice (Fig. 3a; representative scan is shown in ESM Fig. 4). In contrast, the size of the islets (Fig. 3b) and the number of cells per islet (Fig. 3c) remained unchanged. Moreover, no change in the expression of genes involved in beta cell function (insulin, $P d x 1, G c k$ and Glut2 [also known as Slc2a2]) was observed (Fig. 3d). These results showed that chronic treatment of HFD mice with Ki16425 increased the total number of islet cells suggesting that a compensatory behaviour of the endocrine pancreas 
Table 1 Effect of chronic treatment with Ki16425 on HFD-fed mice

\begin{tabular}{lcc}
\hline Variable & Control $(n=6)$ & Ki16425 $(n=6)$ \\
\hline Body weight (g) & $34.3 \pm 2.3$ & $32.4 \pm 1.4$ \\
Body fat mass (\% of total body weight) & $34.1 \pm 0.5$ & $37.4 \pm 1.1$ \\
Body lean mass (\% of total body weight) & $56.6 \pm 0.7$ & $57.7 \pm 0.9$ \\
Subcutaneous adipose tissue (mg) & $969 \pm 27$ & $1,269 \pm 258$ \\
Perigonadal adipose tissue (mg) & $1,711 \pm 198$ & $1,583 \pm 292$ \\
Pancreas (mg) & $249 \pm 32$ & $202 \pm 17$ \\
Liver (mg) & $1,136 \pm 78$ & $1,051 \pm 97$ \\
Gastrocnemius muscle (mg) & $341 \pm 17$ & $359 \pm 19$ \\
Soleus muscle (mg) & $20 \pm 1$ & $19 \pm 2$ \\
Fasted liver triacylglycerols (mg/[g protein]) & $1.51 \pm 0.28$ & $2.07 \pm 0.34$ \\
Fasted muscle triacylglycerols (mg/[g protein]) & $7.55 \pm 0.67$ & $7.92 \pm 1.80$ \\
Fasted muscle glycogen (mg/[g protein]) & $14.7 \pm 3.5$ & $14.3 \pm 2.5$
\end{tabular}

might be responsible for Ki16425-mediated increase in fasting insulinaemia.

Chronic treatment with Ki16425 improves insulin tolerance in HFD mice and increases liver glycogen content To investigate the influence of Ki16425 on glucose homeostasis, insulin tolerance tests were performed in HFD mice. Chronic treatment with Ki16425 led to a significant improvement in insulin tolerance when compared with control vehicle (Fig. 4a, b). The decline in blood glucose concentration between 0 and 60 min following insulin injection was faster in Ki16425treated mice than in vehicle-treated control mice suggesting a better insulin sensitivity. Interestingly, control glycaemia increased again after $60 \mathrm{~min}$, reflecting endogenous glucose production, whereas in Ki16425-treated mice glycaemia stayed at a low level (Fig. 4a). These observations suggest that Ki16425 has an impact on liver glucose metabolism.

To test this hypothesis, livers were examined. Ki16425treated mice showed no change in liver weight (Table 1) or triacylglycerol content (Table 1). In contrast, Ki16425treated mice displayed a significant increase in liver glycogen content when compared with vehicle-treated control mice; this was observed in overnight fasted mice as well as after $18 \mathrm{~h}$ re-feeding (Fig. 4c). Glycogen content results from a balance between synthesis and degradation. Livers from Ki16425-treated mice displayed higher gene expression of Gck (involved in glucose uptake) and lower expression of G6pase (also known as G6pc) and Pepck (also known as $P c k 1$, involved in glucose production) when compared with control mice (Fig. 4d). A trend towards a reduced expression of $G y k$ (involved in neoglucogenesis) was also observed but did not reach significance $(p=0.08)$ (Fig. $4 d)$. Although we are aware of the limitations in the functional conclusions that can be drawn from mRNA measurements, these results suggested that Ki16425-mediated increase in liver glycogen content might result from a reduction in glucose release and an increase in glucose uptake in liver. These changes might explain, at least in part, Ki16425mediated improvement of insulin tolerance and could also
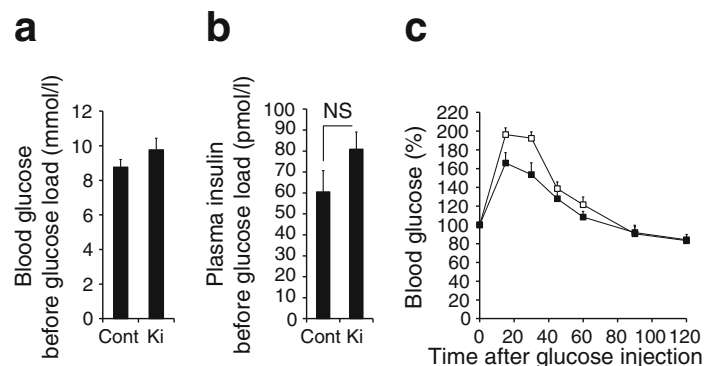

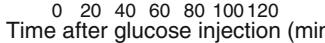

Fig. 2 Chronic treatment with Ki16425 improves glucose tolerance in HFD mice. After 9 weeks of diet, HFD mice received daily injections of Ki16425 (Ki) or vehicle (Cont) for 3 weeks. Mice were fasted for $7 \mathrm{~h}$ before conducting a glucose tolerance test. Blood glucose (a) and plasma insulin (b) concentrations measured before glucose load. (c) Blood glucose concentration during glucose tolerance tests, expressed

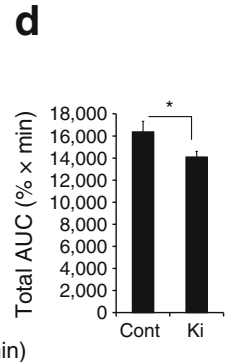

d
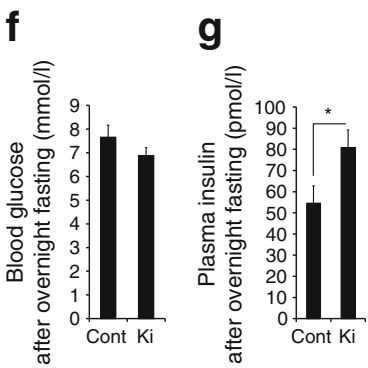

as per cent of concentration at time 0, and corresponding AUC (d). White squares, vehicle; black squares, Ki16425. (e) Fold change in plasma insulin from $15 \mathrm{~min}$ before to $15 \mathrm{~min}$ after glucose load. Blood glucose (f) and plasma insulin (g) concentrations after overnight fasting. Values are means \pm SEM of six to 12 mice per group. NS, $p=0.072 ; * p<0.05$, comparing indicated values 

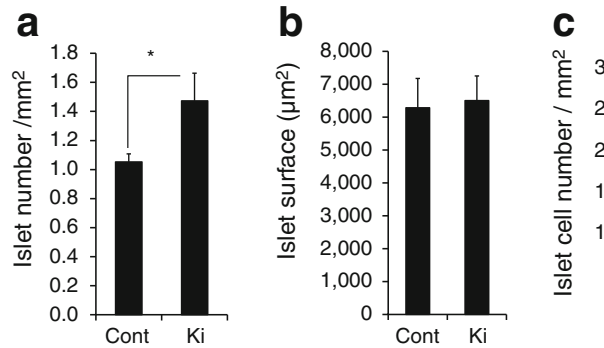

Fig. 3 Chronic treatment with Ki16425 increases islet cell number in HFD mice. HFD mice were treated as described in the legend for Fig. 2, and pancreas was analysed after overnight fasting. Islet number (a), islet surface (b) and number of cells per islet (c) were determined

contribute to Ki16425-mediated improvement in glucose tolerance.

Chronic treatment with Kil6425 increases glucose use in skeletal muscle Since skeletal muscles play a crucial role in glucose homeostasis, they were also examined after chronic treatment with Ki16425. Neither their weight nor their glycogen and triacylglycerol contents were modified (Table 1). Nevertheless, soleus muscle gene expression analysis a

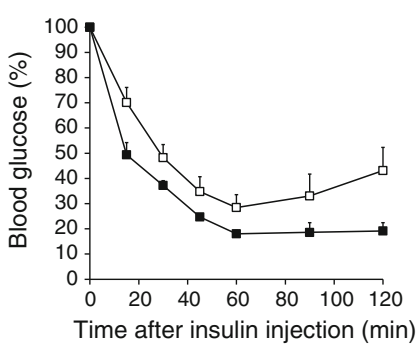

b

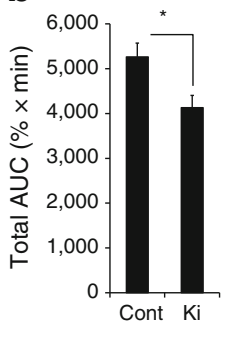

C

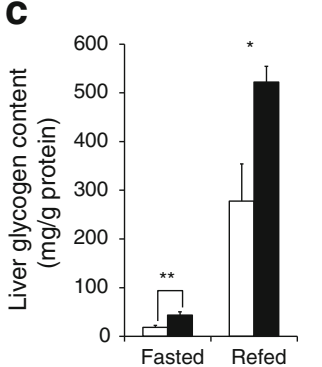

d

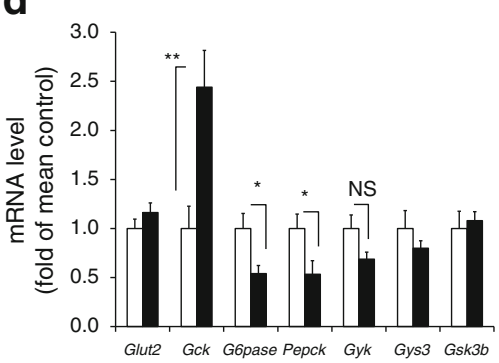

Fig. 4 Chronic treatment with Ki16425 improves insulin tolerance and increases liver glycogen content in HFD mice. HFD mice were treated as described in the legend for Fig. 2, and insulin tolerance tests were conducted after $7 \mathrm{~h}$ fasting. (a) Blood glucose concentration during glucose tolerance tests, expressed as per cent of concentration at time 0 , and corresponding AUC (b). White squares, vehicle; black squares, Ki16425. (c) Glycogen content after overnight fasting (Fasted) and $18 \mathrm{~h}$ after refeeding (Refed). (d) Gene expression of fasted mice. Values are means \pm SEM of six mice per group; white bars, vehicle; black bars, Ki16425. NS, $p=0.08 ;{ }^{*} p<0.05$ and ${ }^{* *} p<0.01$, comparing indicated values d

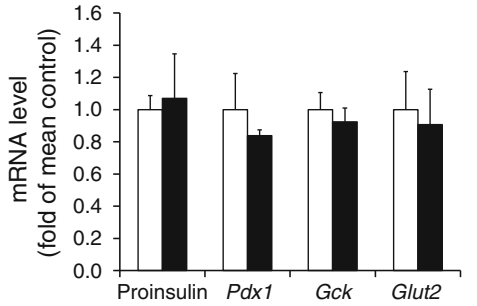

by histomorphology. Representative scans are shown in ESM Fig. 3. (d) Gene expression in whole pancreas from HFD mice; white bars, vehicle; black bars, Ki16425. Values are means \pm SEM of six to 12 mice per group. ${ }^{*} p<0.05$, comparing indicated values

showed a significant increase in Glut1 (also known as Slc2al) and Hk2, but not Glut4 (also known as Slc2a4) mRNAs (Fig. 5a). Although we are aware of the limitations in the functional conclusions that can be drawn from mRNA measurements, these results suggested a change in glucose transport. In ex vivo muscles, a slight increase in 2-deoxy$\mathrm{D}-\left[{ }^{3} \mathrm{H}\right]$ glucose transport was indeed observed, but the difference did not reach significance (Fig. 5b). Nevertheless, a significant increase in ${ }^{14} \mathrm{CO}_{2}$ production from $\left[{ }^{14} \mathrm{C}\right]$ glucose

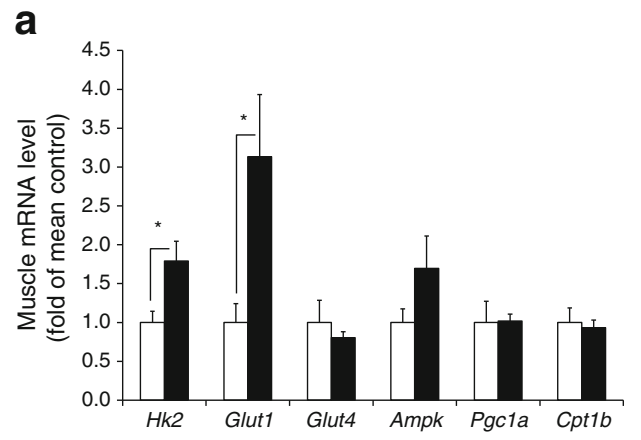

b
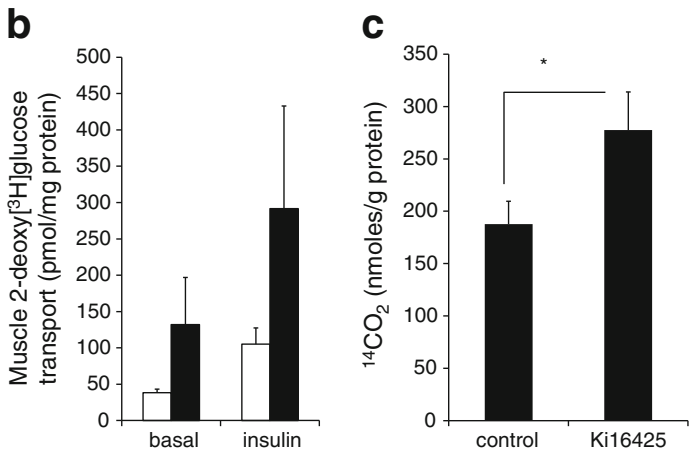

Fig. 5 Influence of mid-term chronic treatment with Ki16425 on glucose metabolism in skeletal muscle of HFD mice. HFD mice were treated as described in the legend to Fig. 2 before quantifying gene expression (a) and measuring 2-deoxy $\left[{ }^{3} \mathrm{H}\right]$ glucose transport (b) and $\left[{ }^{14} \mathrm{C}\right]$ glucose oxidation (c) in ex vivo muscle; white bars, vehicle; black bars, Ki16425. Values are means \pm SEM of six to 12 mice per group. $* p<0.05$, comparing indicated values 
in ex vivo muscle was observed in Ki16425-treated mice when compared with vehicle-treated control mice (Fig. 5c). Although one should remain cautious about extrapolating ex vivo observations to the in vivo situation, our results nevertheless suggested that chronic treatment of HFD-fed mice with Ki16425 could enhance glucose oxidation in muscle and this might contribute, at least in part, to Ki16425mediated improvement in glucose tolerance.

\section{Discussion}

The objective of this work was to study the involvement of LPA in glucose intolerance associated with obesity. We previously reported that transgenic-mediated reduction of LPA synthesis was associated with improved glucose tolerance in HFD-fed mice [19], suggesting that LPA has a negative impact on glucose homeostasis. Here we strengthened this hypothesis by showing the deleterious effect of LPA on glucose tolerance through inhibition of insulin secretion and we demonstrated that chronic pharmacological blockade of LPA receptors with Ki16425 can reverse the deterioration in glucose homeostasis in HFD obese prediabetic mice.

We first showed that acute injection of LPA leads to a strong impairment of glucose tolerance. This observation clearly demonstrates a direct negative influence of LPA on glucose tolerance. This effect is mediated by LPA receptors since the antagonist Ki16425 blocks it. The deleterious effect of LPA was associated with inhibition of glucoseinduced insulin secretion with no change in insulin tolerance. These observations strongly suggest that the acute deleterious impact of LPA on glucose tolerance is mediated by inhibition of insulin secretion rather than by an alteration of insulin sensitivity. Moreover, in vitro experiments show that LPA dose-dependently inhibited glucose-induced insulin secretion, directly on isolated islets. This is not in agreement with the findings of Metz et al [31] who reported that glucose-induced insulin secretion was not influenced by LPA in isolated rat islets. Whereas species differences in islet sensitivity to LPA could explain this discrepancy, the way LPA is presented to the islets should also be taken into account. Whereas in our work, LPA was brought to islets in solution in an albumin-containing buffer, Metz et al used an albumin-free vehicle [31]. The latter is not favourable to LPA solubility or its presentation to receptors [32].

Our data also show that inhibition of glucose tolerance is not limited to exogenous LPA and can be extended to endogenous LPA. LPA plasma concentration is higher in HFD mice than in ND mice, and we found that Ki16425 improves glucose tolerance by itself in HFD mice but not in ND mice. Having demonstrated the deleterious effect of exogenous LPA on glucose tolerance, the most likely explanation is that overproduction of endogenous LPA in HFD mice exerts a tonic inhibitory effect on glucose tolerance that can be alleviated by blocking LPA receptors. Such a conclusion is close to that drawn from our previous report [19] showing that deletion of the LPA-synthesising enzyme autotaxin in adipose tissue was associated with an improvement in glucose tolerance in HFD mice but not in ND mice. Therefore, blocking LPA synthesis or LPA receptors leads to the same metabolic alterations. Increased LPA synthesis in HFD obese mice very likely participates in the deterioration of their glucose tolerance. LPA can therefore be considered to play an important part in the onset of obesity-associated impairment of glucose homeostasis. Thus, autotaxin and LPA receptors represent possible valuable targets for the treatment of metabolic disorders associated with obesity.

We also found that chronic treatment of HFD mice with Ki16425 improved insulin tolerance, increased liver glycogen storage and increased the capacity of muscle to oxidise glucose. These changes are obvious signs of a better disposal of glucose in peripheral organs and are very likely responsible for Ki16425-induced improvement of glucose tolerance. Nevertheless, chronic treatment with Ki16425 also increases fasting insulin. This was initially intriguing to us since increased insulinaemia is usually associated with insulin resistance. It is well established that HFD obesity leads, after a compensatory phase, to progressive beta cell degeneration responsible, at least in part, for the deregulation of insulin production associated with obesity [33]. Histological analysis of the pancreas revealed that the number of pancreatic islets cells was higher in Ki1625treated mice than in vehicle-treated control mice. Assuming that more than $95 \%$ of the cells present in islets are beta cells, it can be concluded that the effect of Ki16425 on the number of beta cells could explain, at least in part, the observed increased in fasting insulin. The influence of Ki16425 on plasma insulin might therefore be interpreted as a protective action against HFD-mediated alteration of beta cells. Nevertheless, we noticed no change in proinsulin mRNA expression, so a possible impact of Ki16425 on the secreting activity of beta cells cannot be excluded. Increased insulin production is recognised as a compensatory mechanism intended to maintain glycaemia when peripheral insulin sensitivity is altered. One can thus propose that the influence of Ki16425 on insulinaemia contributes to Ki16425-mediated improvement in glucose disposal. A possible mechanism could be that increased insulinaemia has an impact on the expression of genes positively involved in glucose homeostasis. This hypothesis is supported by our results showing that Ki16425 treatment affected the expression of several insulin-sensitive genes such as Gck, G6pase and Pepck.

In conclusion, this work presents strong evidence for the role of LPA as a deleterious lipid mediator involved in the deterioration of glucose homeostasis associated with obesity. Moreover, the demonstration of the beneficial effects of the antagonist Ki16425 indicates that LPA receptors might represent potential interesting pharmacological targets to prevent and/or treat the metabolic defects associated with obesity. 
Acknowledgements We thank I. Castan-Laurell, V. Bourlier and C. Moro (Inserm U1048, Toulouse, France) and J. Boucher (Joslin Institute, Harvard University, Boston, MA, USA) for fruitful discussions and reviewing the manuscript. We also thank $\mathrm{F}$. Capilla from the service of Histopathology (Inserm US006 CREFRE, Toulouse, France) for her very helpful technical assistance. M. Prentki is the recipient of the Canada research chair in diabetes and metabolism. C. Rancoule is supported by a grant from the Ministère de l'Education Nationale de la Recherche et de la Technologie (France). C. Attané is supported by a posdoctoral fellowship from the Canadian Diabetes Association.

Funding This work was supported by grants from Inserm (to J. S. Saulnier-Blache) and the Fondation pour la Recherche Médicale (grant no. DRM20101220459) (to J. S. Saulnier-Blache) and the Canadian Institute of Health Research (to M. Prentki).

Duality of interest The authors declare that there is no duality of interest associated with this manuscript.

Contribution statement All authors participated in the conception and design, or analysis and interpretation of the data, contributed to drafting and revising the manuscript, and gave final approval of the version to be published.

\section{References}

1. Lee MJ, Wu Y, Fried SK (2010) Adipose tissue remodeling in pathophysiology of obesity. Curr Opin Clin Nutr Metab Care $13: 371-376$

2. Gutierrez DA, Puglisi MJ, Hasty AH (2009) Impact of increased adipose tissue mass on inflammation, insulin resistance, and dyslipidemia. Curr Diabetes Rep 9:26-32

3. Karastergiou K, Mohamed-Ali V (2010) The autocrine and paracrine roles of adipokines. Mol Cell Endocrinol 318:69-78

4. Choi JW, Herr DR, Noguchi K et al (2010) LPA receptors: subtypes and biological actions. Annu Rev Pharmacol Toxicol 50:157-186

5. Boutin JA, Ferry G (2009) Autotaxin. Cell Mol Life Sci 66:3009-3021

6. Nakanaga K, Hama K, Aoki J (2010) Autotaxin - an LPA producing enzyme with diverse functions. J Biochem 148:13-24

7. Kano K, Arima N, Ohgami M, Aoki J (2008) LPA and its analogs attractive tools for elucidation of LPA biology and drug development. Curr Med Chem 15:2122-2131

8. Im DS (2010) Pharmacological tools for lysophospholipid GPCRs: development of agonists and antagonists for LPA and S1P receptors. Acta Pharmacol Sin 31:1213-1222

9. Rancoule C, Pradere JP, Gonzalez J et al (2011) Lysophosphatidic acid-1-receptor targeting agents for fibrosis. Expert Opin Investig Drugs 20:657-667

10. Ohta H, Sato K, Murata N et al (2003) Ki16425, a subtypeselective antagonist for EDG-family lysophosphatidic acid receptors. Mol Pharmacol 64:994-1005

11. Boucharaba A, Serre CM, Guglielmi J, Bordet JC, Clezardin P, Peyruchaud O (2006) The type 1 lysophosphatidic acid receptor is a target for therapy in bone metastases. Proc Natl Acad Sci U S A 103:9643-9648

12. Pradere JP, Klein J, Gres S et al (2007) LPA1 receptor activation promotes renal interstitial fibrosis. J Am Soc Nephrol 18:3110-3118

13. Zhou Z, Subramanian P, Sevilmis G et al (2011) Lipoproteinderived lysophosphatidic acid promotes atherosclerosis by releasing CXCL1 from the endothelium. Cell Metab 13:592-600

14. Valet P, Pages C, Jeanneton O et al (1998) Alpha2-adrenergic receptormediated release of lysophosphatidic acid by adipocytes. A paracrine signal for preadipocyte growth. J Clin Invest 101:1431-1438
15. Gesta S, Simon MF, Rey A et al (2002) Secretion of a lysophospholipase D activity by adipocytes: involvement in lysophosphatidic acid synthesis. J Lipid Res 43:904-910

16. Ferry G, Tellier E, Try A et al (2003) Autotaxin is released from adipocytes, catalyzes lysophosphatidic acid synthesis, and activates preadipocyte proliferation. Up-regulated expression with adipocyte differentiation and obesity. J Biol Chem 278:18162-18169

17. Boucher J, Quilliot D, Praderes JP et al (2005) Potential involvement of adipocyte insulin resistance in obesity-associated upregulation of adipocyte lysophospholipase $\mathrm{D} /$ autotaxin expression. Diabetologia 48:569-577

18. Rancoule C, Dusaulcy R, Treguer K et al (2012) Depot-specific regulation of autotaxin with obesity in human adipose tissue. $\mathrm{J}$ Physiol Biochem 68:635-644

19. Dusaulcy R, Rancoule C, Gres S et al (2011) Adipose-specific disruption of autotaxin enhances nutritional fattening and reduces plasma lysophosphatidic acid. J Lipid Res 52:1247-1255

20. Simon MF, Daviaud D, Pradere JP et al (2005) Lysophosphatidic acid inhibits adipocyte differentiation via lysophosphatidic acid 1 receptor-dependent down-regulation of peroxisome proliferatoractivated receptor gamma2. J Biol Chem 280:14656-14662

21. Coy PE, Taneja N, Lee I, Hecquet C, Bryson JM, Robey RB (2002) LPA is a novel lipid regulator of mesangial cell hexokinase activity and HKII isoform expression. Am J Physiol Ren Physiol 283:F271-F279

22. Keller JN, Steiner MR, Mattson MP, Steiner SM (1996) Lysophosphatidic acid decreases glutamate and glucose uptake by astrocytes. J Neurochem 67:2300-2305

23. Yea K, Kim J, Lim S et al (2008) Lysophosphatidic acid regulates blood glucose by stimulating myotube and adipocyte glucose uptake. J Mol Med 86:211-220

24. Fan H, Zingarelli B, Harris V, Tempel GE, Halushka PV, Cook JA (2008) Lysophosphatidic acid inhibits bacterial endotoxin-induced pro-inflammatory response: potential anti-inflammatory signaling pathways. Mol Med 14:422-428

25. Hashimoto T, Ohata H, Momose K (2004) Itch-scratch responses induced by lysophosphatidic acid in mice. Pharmacology 72:51-56

26. Subramanian P, Karshovska E, Reinhard P et al (2010) Lysophosphatidic acid receptors LPA1 and LPA3 promote CXCL12-mediated smooth muscle progenitor cell recruitment in neointima formation. Circ Res 107:96-105

27. Saulnier-Blache JS, Girard A, Simon MF, Lafontan M, Valet $P$ (2000) A simple and highly sensitive radioenzymatic assay for lysophosphatidic acid quantification. J Lipid Res 41:1947-1951

28. Nahle Z, Hsieh M, Pietka T et al (2008) CD36-dependent regulation of muscle FoxO1 and PDK4 in the PPAR delta/beta-mediated adaptation to metabolic stress. J Biol Chem 283:14317-14326

29. Dray C, Knauf C, Daviaud D et al (2008) Apelin stimulates glucose utilization in normal and obese insulin-resistant mice. Cell Metab 8:437-445

30. Peyot ML, Guay C, Latour MG et al (2009) Adipose triglyceride lipase is implicated in fuel- and non-fuel-stimulated insulin secretion. J Biol Chem 284:16848-16859

31. Metz SA (1986) Lysophosphatidylinositol, but not lysophosphatidic acid, stimulates insulin release. A possible role for phospholipase A2 but not de novo synthesis of lysophospholipid in pancreatic islet function. Biochem Biophys Res Commun 138:720-727

32. Hama K, Bandoh K, Kakehi Y, Aoki J, Arai H (2002) Lysophosphatidic acid (LPA) receptors are activated differentially by biological fluids: possible role of LPA-binding proteins in activation of LPA receptors. FEBS Lett 523:187-192

33. Nolan CJ, Damm P, Prentki M (2011) Type 2 diabetes across generations: from pathophysiology to prevention and management. Lancet 378:169-181 